\title{
The COVID 19 Related Increased Negative Impact of the Unmonitored Use of Digital Technology on Children in KSA
}

\author{
Maram Aloufi ${ }^{1}$, Nouf Alsulami ${ }^{1}$, Layan Alqahtani ${ }^{1}$, Joud Baali ${ }^{1}$, Mashael Khayyat ${ }^{1 *}$ \\ ${ }^{\mathrm{T}}$ Department of Information Systems and Technology, College of Computer Science and Engineering, University of \\ Jeddah, Jeddah, Saudi Arabia
}

*Corresponding Author: Mashael Khayyat (mkhayyat@uj.edu.sa)

\section{Article History}

Received: 15.12 .2021

Accepted: 22.01 .2022

Published: 27.01.2022

\begin{abstract}
The use of digital technology can become a behavioral addiction that negatively affects a person's social and academic life. Nowadays, children all over the world are becoming addicted to technologies - more so than in any past generations. Digital technology has become an integral part of everyday life, and children use it to study and play. Also, some parents use digital technology as a 'babysitter' to entertain their children so that the parents can do other things. This affects children's brains and their social, emotional, cognitive, and physical development. This research aims to assess the potential impact of technology on children's behavior. To collect data, this paper used an online survey created with Google Forms. The sample targeted parents. There were 207 responses. The results of the questionnaire and previous studies conducted on the effect of technology on children taken from scientific research found that as technology use increases, the effect it can have on brain function increases. This effect has been linked to physical and psychological problems such as reduced attention span, risk of depression, obesity, and bullying. Based on the study's results it is recommended that parents monitor their children's use of digital technology; this includes setting a time limit for using devices, monitoring content, blocking violent and intimidating games for children, and setting a bedtime. In addition, apps such as Qustodio, Norton Family Premier, and Amazon Free Time are suggested as tools to support parents in overseeing their children's use of digital technology.
\end{abstract}

Keywords: Children, Technology, Impact, Parents, Devices, Applications.

\section{INTRODUCTION}

Technological devices are becoming vital aspects of our daily routines. Computers, cellphones, other digital devices have grown in importance as a source of activity and a means of communication [1, 5]. To a teenager today, a world without smartphones or the Internet may be difficult to imagine, simply because they were born in an era where everything has become technical, including education [5]. This is even more true for younger children, who grow up in a world where technology is everywhere, from reading an e-book on a tablet to tackling a crossword problem with a buddy from another country [4-6, 10]. Children's violence and brutality, erroneous perceptions of the world, and difficulties communicating with others are all examples of computers' harmful effects on children [3, 5, 10, 12]. Furthermore, during the COVID-19 epidemic, schools turned online learning, resulting in an increase in the number of hours children are exposed to technology each day [5]. In addition, children are turning to social media and video games for entertainment $[3,7]$

\section{LITERATURE REVIEW}

The influence of children's use of technologies on their development is still controversial. Some research proposes that technologies have impeded children's social, emotional, physical, and cognitive development. Some early research focused on the effect of watching television on children and their overall psychology, including impulsive behavior [5]. Excessive screen exposure and less sleep negatively impacts a child's life. Children who use electronic devices in the evening hours show significantly shorter nighttime sleep duration than those with no evening screen

Copyright (C) 2022 The Author(s): This is an open-access article distributed under the terms of the Creative Commons Attribution 4.0 International License (CC BY-NC 4.0) which permits unrestricted use, distribution, and reproduction in any medium for non-commercial use provided the original author and source are credited.

CITATION: Maram Aloufi, Nouf Alsulami, Layan Alqahtani, Joud Baali, Mashael Khayyat (2022). The COVID 19 
exposure. In addition, the more TV children watch, the more likely they are to be obese; therefore, researchers have hypothesized that three different mechanisms play a role: increased caloric intake while engaging with TV or caused by advertising, reduced resting metabolism, and displacement of physical activity $[4,11]$. Obese children have a $54 \%$ higher rate of absenteeism from school than their normal-weight counterparts and have poorer levels of academic achievement [11]. Although research demonstrates that well-designed, age-appropriate, educational television can be beneficial to children of preschool age, studies on infants and toddlers suggest that these young children may better understand and learn from real-life experiences than they do from video $[10,12]$.

Unfortunately, COVID-19 has caused the need for social distancing, which has caused an increase in the use of technology among teenagers and children, and has had a negative impact on their lives. Excessive use of technology leads to wasting their time with something unhelpful [7]. Studies have also shown a strong relationship between violence in games and actual violence and that these games lead to social isolation and lack of communication with others. It has also been associated with children's lack of attention, aggression, lack of physical activity, and sleep problems. In a study looking at the effect of television on quality of sleep-in children, $43 \%$ of children in the survey were abnormal. Children who watch television are also more likely to have problems paying attention, acting aggressively, and getting enough sleep [7].

In a study of preschool children, $43 \%$ of children with a television set in their bedroom had abnormal sleep behaviors, notably greater scores in sleep terrors, nightmares and sleep talk [1-3]. The American Academy of Pediatrics and the Canadian Society of Pediatrics states babes aged 0-2 years should not have any exposure to technology to one hour per day and 6-18-year-old to 2 hours per day. Overuse of digital technology has been associated with rapid brain development, negatively associated with increased executive functioning, attention deficit, and learning impairment. Addiction, lack of care, and supervision from parents can lead to children's addiction to devices and aggression; violent content that children watch can cause a high rate of physical violence [9].

\section{METHODOLOGY}

This paper used an online survey created with Google Forms to collect primary data. The survey instrument consisted of 17 questions designed to find the impact of technology on children. Criteria for inclusion was being a parent. 207 responses have been received and analyzed.

\section{RESULTS}

Significantly, about $63.7 \%$ of the participating parents agreed that the devices kept the child away from eating meals regularly (see Figure 1). Furthermore, almost half $(49.5 \%$ ) of the parent respondents reported that technology harms the child in terms of study or educational attainment (see Figure 2).

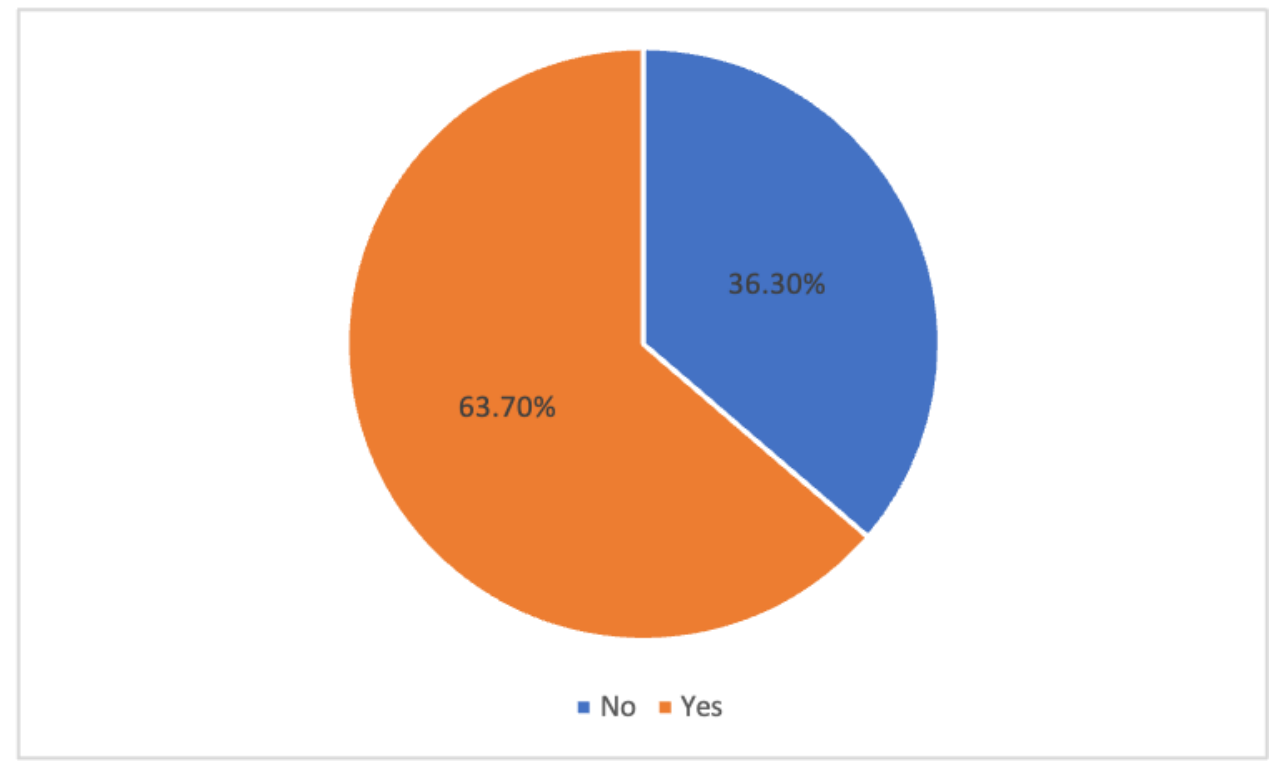

Fig 1: The impact of children's use of devices and the delay in eating their meals 


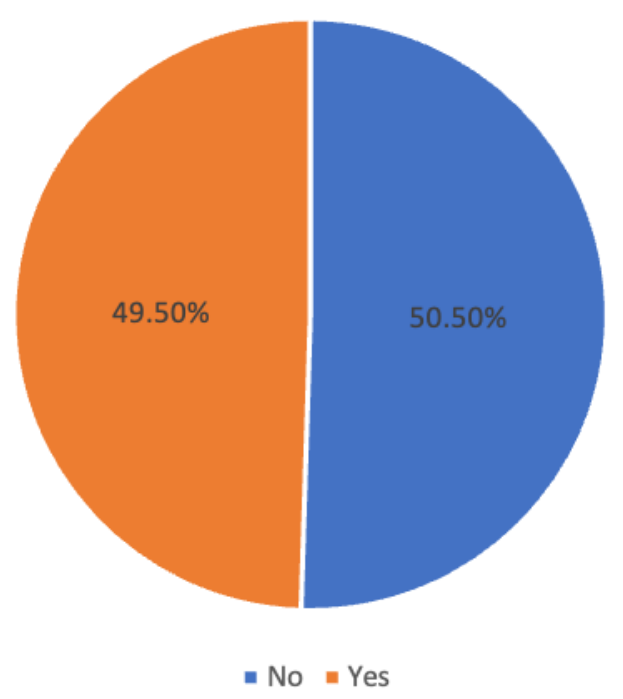

Fig 2: The effect of devices on children's academic achievement

About $85.4 \%$ agreed that their children had become addicted to these devices, whereas $14.5 \%$ did not notice this addiction (see Figure 3).

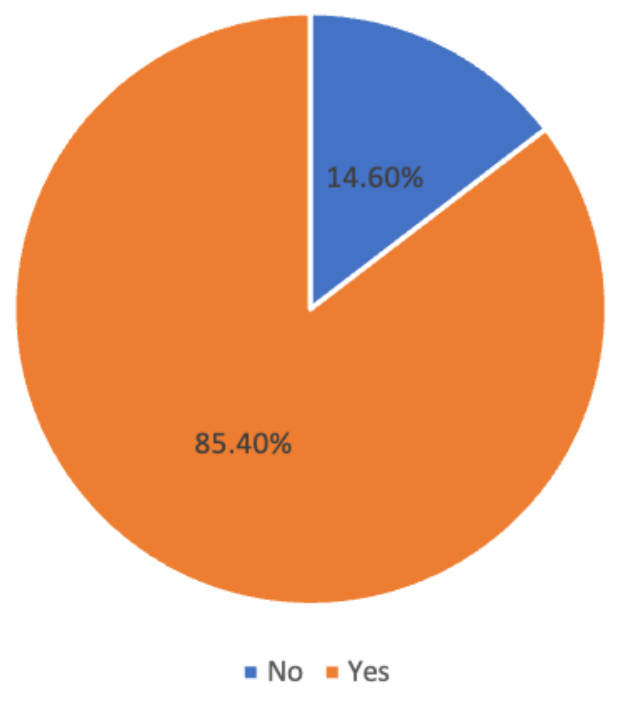

Fig 3: How addictive children are to devices

$67.8 \%$ of parents agreed that the Corona pandemic has played a significant role in increasing children's addiction to devices, Figure 4 illustrates parents' opinions on how the Corona pandemic affected their children using devices. 


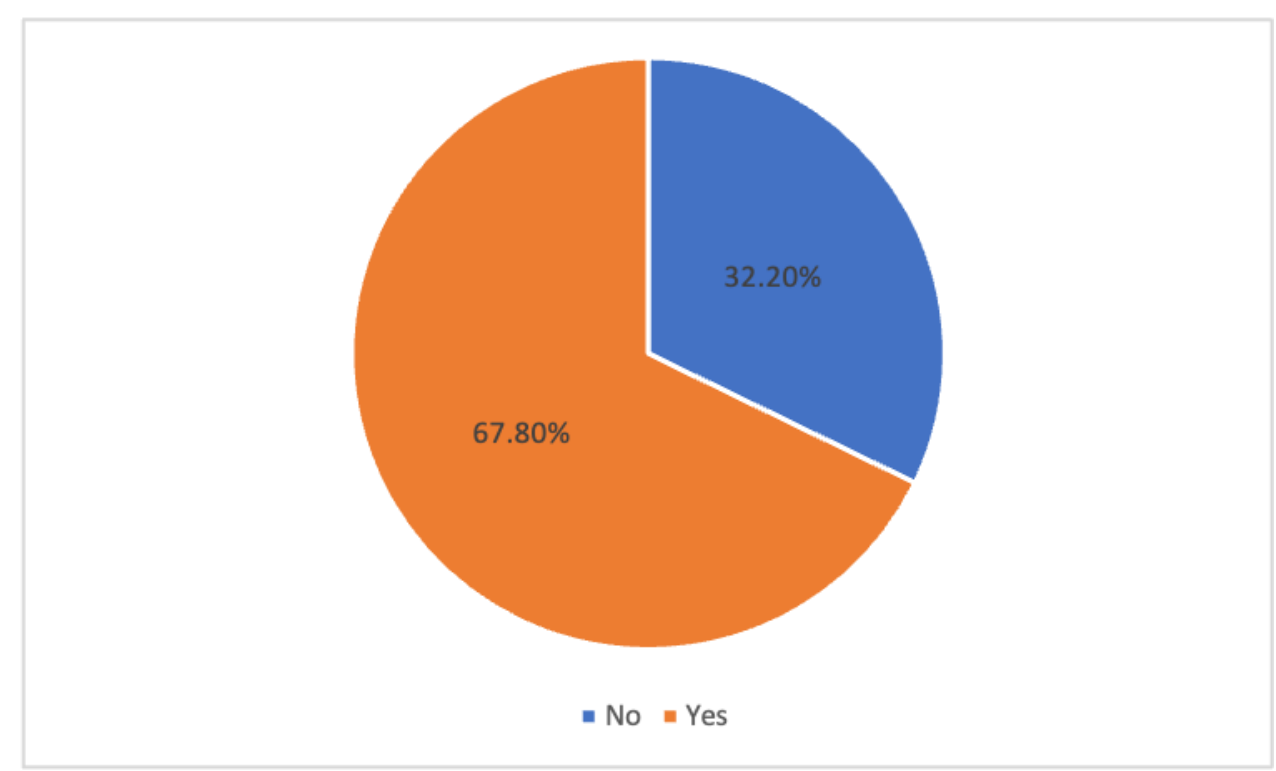

Fig 4: The impact of the Corona pandemic on children

The Corona pandemic affected children in terms of their increased use of devices by $67.8 \%$; among the reasons noted were closing entertainment centers for children, lack of social events, shifting to distance education, quarantine, and staying at home,

Noted impacts were:

- Became addicted to using the devices.

- Increased time of using devices.

- The transferer from traditional to online education

- The quarantine increased the amount of free time at home.

- A large percentage $(88.2 \%)$ of parents did not use an application to assign time and content to their children; those who used an application accounted for $11.8 \%$ (see Figure 5).

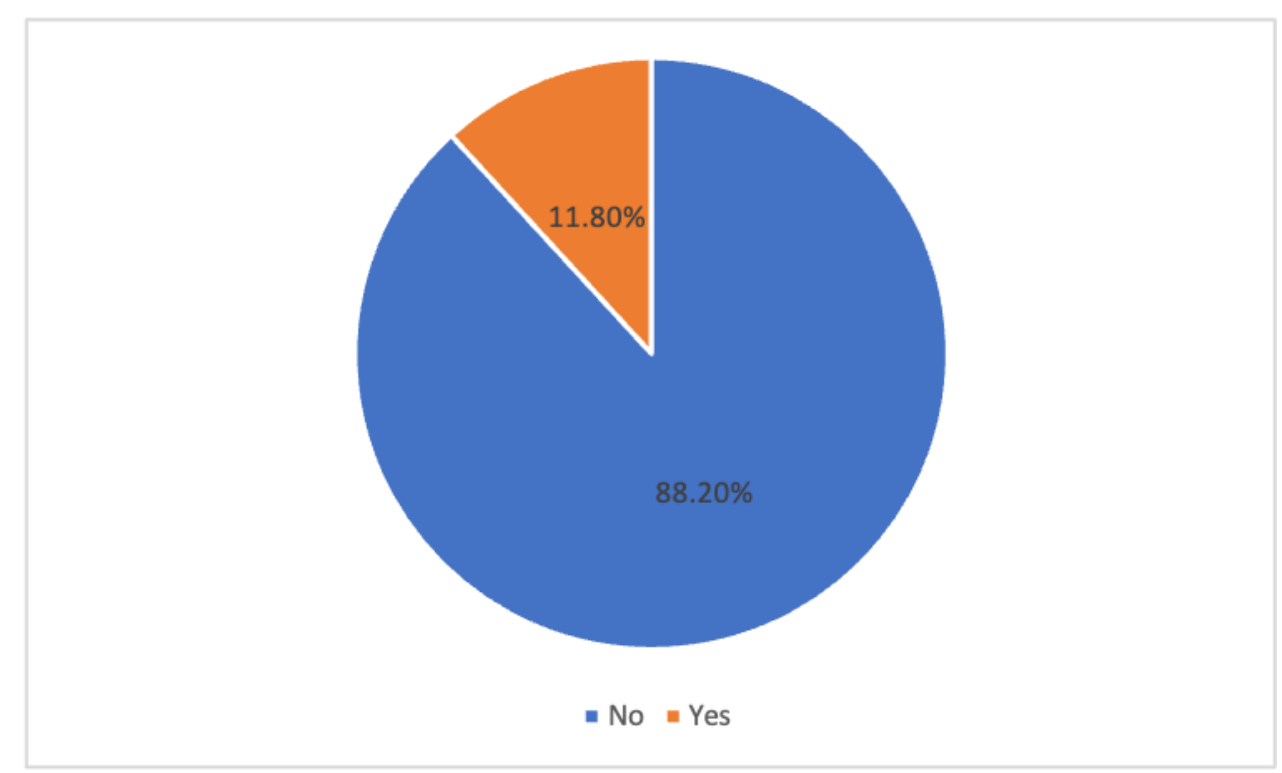

Fig 5: If parents use an app to set time and content for their children

\section{DISCUSSION}

Initially several studies in this research have been used to support the negative aspects of technology's impact on children. A lack of parental control over the content and time can result in the children becoming addicted to technology and spending most of their time on it. This problem leads to health and psychological issues such as obesity and it is necessary to educate the parents of children about the dangers of technology to children. 


\section{CONCLUSION}

The purpose of this paper was to raise the awareness of the impact of this technology on children. The problems can be summarized under five broad headings:

First: The use of technology in an inappropriate manner in terms of duration and being sedentary may cause health and physical problems such as problems in the bones and muscles, obesity, and lack of growth.

Second: Children's increased use of technology may lead to isolation and difficulty communicating with others.

Third: Children's increased use of technology may affect their brains socially, emotionally, and cognitively.

Fourth: The presence of the TV, computer, and mobile phone in the children's bedroom cause them to sleep less and be lethargic.

Fifth: Children's increased use of technology may lead to physical and psychological problems such as reduced attention span, risk of depression, and obesity.

Based on the study, the following recommendations for parents on how to minimize the negative effects of technology on children are made:

- Limiting usage to child trusted video channels rather than restricting all video viewing, with a specific number of hours per day, as a way to reduce the negative effects of technology on children.

- Prohibiting children's games that are violent or disturbing.

- Explaining the difference between fantasy and reality to decrease the negative emotional impact on children,

- Using parental control software to monitor what the child watches and plays online.

Using applications to limit usage and restrict content access such as (Google Family Link, Amazon Free time, Norton Family Primer, Kid Logger \& Custodia).

Finally, we must acknowledge that technology has become a vital part of our life that we cannot live without, On the other hand, we have become slaves to these digital gadgets, but we can prevent its potential downsides after learning about its impact on children. Reasonable content use and regulation will ensure that children get the most out of technology while avoiding its drawbacks.

\section{REFERENCES}

1. Mustafaoğlu, R., Zirek, E., Yasacı, Z., \& Özdinçler, A. R. (2018). The negative effects of digital technology usage on children's development and health. Addict: the Turkish journal on addictions, 5(2), 13-21.

2. Almuaigel, D., Alanazi, A., Almuaigel, M., Alshamrani, F., AlSheikh, M., Almuhana, N., ... \& Mansi, K. (2021). Impact of Technology Use on Behavior and Sleep Scores in Preschool Children in Saudi Arabia. Frontiers in psychiatry, 12, 601 .

3. Limone, P., \& Toto, G. A. (2021). Psychological and Emotional Effects of Digital Technology on Children in COVID-19 Pandemic. Brain Sciences, 11(9), 1126.

4. Gottschalk, F. (2019). Impacts of technology use on children: Exploring literature on the brain, cognition, and wellbeing.

5. Samhan, B., \& Ruane, R. (2021). Exploring the Potential Effects of Technology Exposure on Temporary Impulsive Behaviors in Children. Health Behavior and Policy Review, 8(4), 305-318.

6. Grose, M. (2013). The good and the bad of digital technology for kids.

7. Drouin, M., McDaniel, B. T., Pater, J., \& Toscos, T. (2020). Parents and their children used social media and technology at the beginning of the COVID-19 pandemic and associated with anxiety. Cyberpsychology, Behavior, and Social Networking, 23(11), 727-736.

8. Rowan, C. (2014). 10 reasons why handheld devices should be banned for children under the age of 12 . Huffington Post, 9.

9. Al-Hawamdeh, M. A., \& Nawas, M. K. A. (2020). ? Are Kids Mentally Smart or Because of Technology: هل الأطفال مجلة العلوم التربوية و النفسية .أذكياء بطبيعتهم أم بسبب التكنولوجيا التي يستخدمونها؟ 4(9), 160-145.

10. Murtaza, S. A. (2017). Digital heroin the impact of digital gadgets on developing minds an empirical study on growing children of Lahore. In International Conference on Management, Business \& Technology (ICMBT) (pp. 303-309).

11. Wolf, C., Wolf, S., Weiss, M., \& Nino, G. (2018). Children's environmental health in the digital era: understanding early screen exposure as a preventable risk factor for obesity and sleep disorders. Children, 5(2), 31.

12. Al Majali, S. (2020). The digital world for children and its relationship with personality disorders: Exploring emerging technologies. International Journal of Emerging Technologies in Learning (IJET), 15(1). 